\title{
Conditioned taste aversion produced by the oral ingestion of ethanol in the rat
}

\author{
MICHAEL J. ECKARDT \\ University of Oregon Health Sciences Center, Portland, Oregon 97201
}

\begin{abstract}
A taste-aversion paradigm was used to demonstrate that aversive consequences accompany the rapid oral ingestion of $5 \%(\mathrm{v} / \mathrm{v})$ ethanol solutions. The learned taste aversion resulted from five self-administrations of alcohol mixed with an originally preferred flavor at a dosage of $1.51 \mathrm{~g} \mathrm{alcohol} / \mathrm{kg}$ body weight/day. Ingestion of larger doses of alcohol by control animals, however, did not produce any evidence of taste aversion.
\end{abstract}

Although animals can be maintained on alcohol solutions above $5 \%-7 \%(\mathrm{v} / \mathrm{v})$ when they are the sole sources of fluid, animals have not continued to select those solutions when alternative fluids such as water have been made available (Lester, 1966; Mardones, 1960; Myers, 1966; Richter, 1953).

Some experimenters have concluded that the smell and taste of alcohol are aversive to the animal and therefore function to prevent ingestion of large amounts of these solutions (Lester, 1966; Myers \& Veale, 1972). In addition, Lester, Nachman, and LeMagnen (1970) have suggested that the centrally mediated concomitants of intoxication or the condition of inebriation and its accompanying manifestations, e.g., loss of balance, are aversive to the rat. Animals do appear inebriated after drinking alcohol solutions, and it seems reasonable to assume that aversive central effects must be present with this method of administration. Thus, in addition to aversive tastes and smells, another possible explanation of why animals do not preferentially drink large amounts of alcohol solutions is that the centrally mediated systemic effects are aversive.

A procedure that has often been used to demonstrate that a drug has aversive consequences accompanying its administration and/or that subsequent pharmacological effects are aversive is the taste-aversion paradigm (Berger, 1972). Operationally, a drug is administered following the ingestion of a distinctively flavored food or fluid. The drug is defined as being aversive if, after it has been paired with a specific fluid, the subsequent consumption of that fluid is decreased. Therefore, it seems plausible that the oral consumption of alcohol will result in conditioned taste aversion because of its aversive orosensory or aversive central effects, or both.

In opposition to the above reasoning is the hypothesis

This research was supported by Training Grant MH 12640 and Grant MH 1-R01-AA01229 from the National Institute on Alcohol Abuse and Alcoholism and Grant MH 23607 from the National Institutes of Health. This study was part of a doctoral dissertation submitted to the Department of Medical Psy chology, University of Oregon Medical School. Dr. Judson S. Brown is acknowledged for his assistance in the design and interpretation of this experiment. Present address: Psychobiology Research Laboratory, Veterans Administration Hospital, Sepulveda, California 91343 . advanced by Lester et al. (1970) that the oral ingestion of alcohol does not have accompanying aversive central consequences because not enough alcohol is consumed "volitionally under self-selection conditions." This conclusion was based on their failure to obtain conditioned taste aversion when alcohol was intubated into the stomach at a dosage of $2.94 \mathrm{~g} / \mathrm{kg}$. Although an intubated dose of $5.15 \mathrm{~g} / \mathrm{kg}$ did result in a conditioned taste aversion, these authors considered it unlikely that such high dosages would be voluntarily ingested by rats. Lester et al. (1970) noted that their experimental animals appeared inebriated shortly after alcohol was introduced directly into the vascular system, whereas the intubation of alcohol into the stomach resulted in a delayed onset of inebriation. Furthermore, they suggested that the quick onset of the central effects of alcohol with IP injection or intracardiac infusion was correlated with the subsequently detected conditioned aversion. In contrast, the inebriation observed with the intubation of alcohol into the stomach, perhaps because of its siow onset, did not result in conditioned aversion, except at high doses. Kalant (1971) has documented that blood alcohol levels increase at a reduced rate when alcohol is intubated into the stomach because the drug is absorbed primarily from the small intestine.

The present experiment was designed to determine whether conditioned taste aversion to a Kool-Aid flavor could be produced by the oral ingestion of a $5 \%(\mathrm{v} / \mathrm{v})$ alcohol-Kool-Aid combination. The method of administration was to allow the animal to drink the alcohol solution as its sole source of fluid during the conditioning sessions. This differs from the stomach intubation procedure used by Lester et al. (1970) which, as noted above, failed to produce a conditioned taste aversion, except at very high doses. In addition, it seemed likely that five pairings of ethanol with a distinctively flavored solution would be more likely to produce a conditioned aversion than the single pairing used by Lester et al. (1970). Also, the sensitive two-flavor preference-drinking test (Dragoin, McCleary, \& McCleary, 1971; Grote \& Brown, 1971) was used in addition to the single-flavor forced-drinking test 
Table 1

Treatments Administered During the Conditioning Sessions

\begin{tabular}{|c|c|c|}
\hline & \multirow{2}{*}{$\begin{array}{l}\text { Sub- } \\
\text { group }\end{array}$} & Conditioning Days \\
\hline & & $\begin{array}{llllllllll}12 & 13 & 14 & 15 & 16 & 17 & 18 & 19 & 20 & 21\end{array}$ \\
\hline Experimental Groups & $\begin{array}{l}\text { A } \\
\text { B }\end{array}$ & 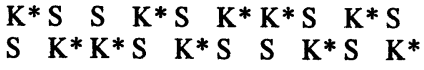 \\
\hline Control Groups & $\begin{array}{l}\text { A } \\
\text { B }\end{array}$ & 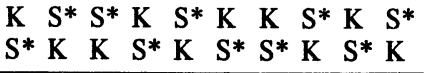 \\
\hline
\end{tabular}

Note-An "*" indicates the presence of $5 \%$ ethanol, $K$ indicates the preferred flavor of Kool-Aid, and $S$ indicates sugar water.

employed by Lester et al. (1970) since it offered increased promise of detecting low levels of conditioned aversion.

\section{METHOD}

\section{Subjects}

The subjects were 20 naive Sprague-Dawley-derived female albino rats from Carworth Farms, Inc., Portage, Michigan.

\section{Procedure}

The subjects were randomly assigned to four groups of five members each and were 110 days of age at the beginning of the experiment. Throughout the study, and for 60 days prior to the start of the experiment, they were housed in a normal 12-h day-night cycle room. An ad-lib food and water regimen was in force during this 60 -day period, after which the animals were housed individually and placed on a fluid-deprivation schedule that permitted $10 \mathrm{~min}$ of drinking per day at the same time each day during the light cycle. For the first 3 days of fluid deprivation, the 10-min drinking sessions (water from a single drinking tube) were followed by 2-min handling and taming sessions for each animal.

Each day thereafter, the animals were weighed and permitted to drink fluid for $10 \mathrm{~min}$ from either of two drinking tubes that projected through the mesh fronts of the cages on the right-front and middle-front areas. Food was available ad lib throughout the experiment from containers mounted against the left front of the cages.

On Days 1 through 6 of the experimental schedule, water was presented for $10 \mathrm{~min}$ in one of the two drinking tubes, its position being alternated daily. Animals were accustomed to the Kool-Aid solutions by allowing them to drink grape-flavored Kool-Aid from one tube and orange-flavored Kool-Aid from the other on Day 7. The formula was .25 teaspoon Kool-Aid, 1.5 teaspoons sugar, and 1.5 cups of water at room temperature. This and other solutions were made up daily. The positions of the tubes were switched half way through the drinking session. On Day 8, the animals were given water (the position of the full tube being varied from where it had been on the previous water-drinking day), and on Days 9 and 10 the two Kool-Aid flavors were offered simultaneously to determine flavor preferences (positions of the flavors were alternated on the 2nd day). Fluid consumptions were recorded, and the flavor with the highest 2-day total was designated the "preferred flavor." This flavor was used throughout the subsequent conditioning period. Water drinking was permitted on Day 11 .

The procedures employed during the conditioning sessions, which were conducted on Days 12 through 21 , are shown in Table 1. During this time, each animal was permitted to drink a designated fluid for $10 \mathrm{~min} /$ day. The only differences between the experimental and control groups were that experimental subjects had 5\% ethanol in the Kool-Aid sessions only, while the control animals had 5\% ethanol in the sugar water sessions only. The experimental and control groups were each divided into two subgroups. As shown in Table 1, animals in Experimental Subgroup A were presented either Kool-Aid (K) or sugar water
(S) in a KSSKSKKSKS order, whereas Experimental Subgroup B animals were subjected to the counterbalanced order of SKKSKSSKSK. The animals in the control subgroups were similarly presented solutions in a counterbalanced order. The procedure of allowing the control animals to ingest alcohol served to control for nonassociative effects, provided the controls ingested at least as much alcohol as the experimental animals. The sugar water solutions used during the conditioning period were made identical to the Kool-Aid formula with the exception that no Kool-Aid was added. The $5 \%$ alcohol solutions were made by adding either Kool-Aid or sugar water to $100 \%$ ethanol.

The conditioning period was followed by 1 day of water drinking. Sixteen days of 10-min preference testing followed to determine whether conditioned aversion had been produced by the experimental treatment. During this period, the positions of the two flavors were alternated daily.

Preference values were calculated for each 2-day period during the preconditioning and postconditioning sessions. These were determined by dividing the total amount of the preferred flavor of Kool-Aid consumed over the 2 -day period by the total intake for the same period. The resulting ratios reflect the animal's Kool-Aid preference over the 2-day period. These ratios are independent of the absolute amount of fluid consumed, being a relative rather than an absolute measure.

Position preference has been demonstrated to be a significant source of bias in experiments in which drinking is measured (Gillespie \& Lucas, 1957). Position preference effects in this experiment were controlled by alternating daily the position(s) of the specified solution(s) and by computing preference ratios on the basis of 2 days' consumptions.

\section{RESULTS}

Daily intakes were recorded in milliliters. Preference ratios for each 2-day period were determined and constituted the data that were analyzed for the preconditioning and postconditioning periods. The amounts consumed each day of Kool-Aid, Kool-Aid $+5 \%$, sugar water, and sugar water $+5 \%$ constituted the data analyzed for the conditioning period.

There were no demonstrable differences between the performances of the two experimental subgroups given counterbalanced orders as measured by preconditioning preference ratios, by first 2-day block of postconditioning preference ratios, or by average daily grams alcohol per kilogram body weight self-administered during the conditioning sessions. The two control subgroups likewise performed identically. Hence, the subgroups of the two sets were combined for all further analyses.

In addition, there were no significant differences between the experimental and control groups on the preconditioning preference values. Hence, subsequent changes in preference between the groups cannot be ascribed to differences existing prior to conditioning.

Two possible measures of conditioned aversion were evaluated. The first was based on the amount of fluid ingested during the forced-drinking regimen in the conditioning sessions. The response of turning away or of stopping drinking the Kool-Aid $+5 \%$ during the first conditioning session should be reinforced by the 
cessation or relative reduction of primary aversive stimulation. Similar responses during subsequent conditioning sessions should also serve to reduce primary aversive stimulation as well as to reduce the effect of any learned aversion to the alcohol solution which might have resulted from the preceding conditioning sessions. On the basis of this formulation, one might expect to observe a progressive reduction in daily intake during the conditioning period for any solution containing alcohol.

The amounts of fluids consumed during the conditioning period by the experimental and control groups are shown in Figure 1. The mean of the two experimental (E) curves (sugar water and Kool-Aid $+5 \%$ ) did not differ significantly from the mean of the two control (C) curves (Kool-Aid and sugar water $+5 \%$ ). A two-factor analysis of variance with repeated measures over drinking opportunities demonstrated that the groups did differ, however, in the quantities of specific solutions consumed during the conditioning period $(\mathrm{F}=94.11, \mathrm{df}=3 / 36, \mathrm{p}<.01)$. Newman-Keuls follow-up tests indicated that the experimental group drank less Kool-Aid $+5 \%$ than the control group drank of Kool-Aid alone $(\mathrm{p}<.01)$, while the control group drank less sugar water $+5 \%$ than the experimental group drank of sugar water alone $(p<.01)$. The data depicted in Figure 1 appear to be consistent with the conclusion that rats prefer sugar water to Kool-Aid. Even though this conclusion is supported by the observation that more sugar water $+5 \%$ was ingested than Kool-Aid $+5 \%(p<.05)$,

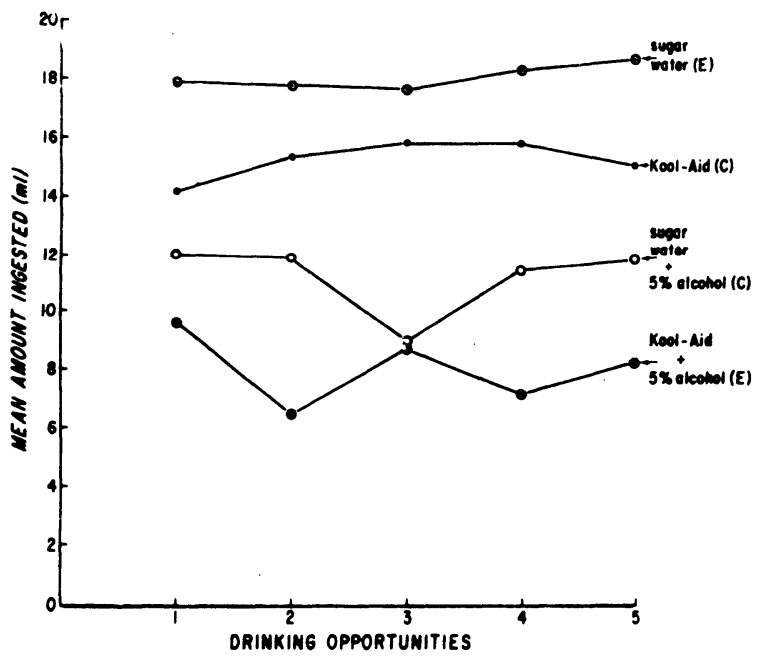

Figure 1. Amounts of fluid consumed during the conditioning period. The number of times an animal was permitted to drink a particular solution is indicated on the abscissa. The data for the experimental group (E) consisted of mean in takes of sugar water and of Kool-Aid $+5 \%$ alcohol, while the data for the control group (C) consisted of the intakes of Kool-Aid and of sugar water $+5 \%$ alcohol. A particular point on the graph represents the mean for both subgroups even though they drank their designated fluids on different days.

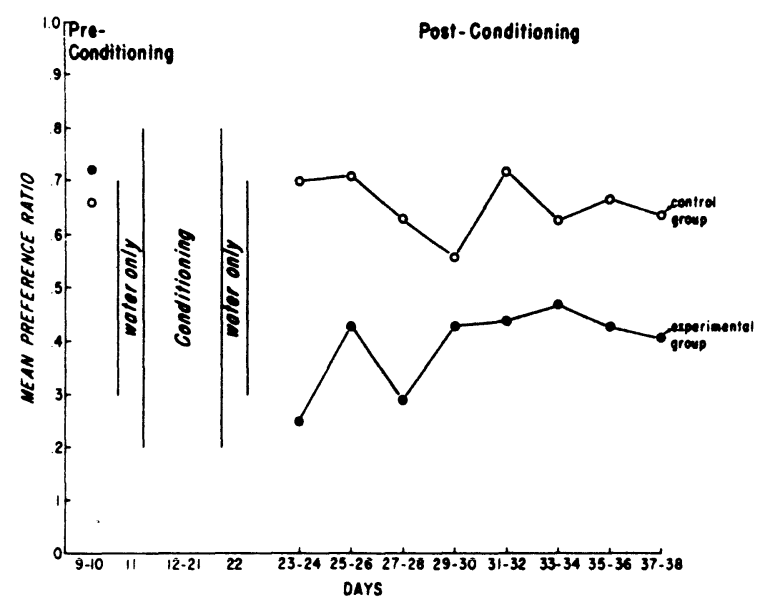

Figure 2. The mean preference ratio (proportion of preferred flavor of Kool-Aid to total fluid intake) for each 2-day block during the preconditioning and postconditioning sessions. The Kool-Aid solutions did not contain alcohol.

no statistical difference was discernible between ingested amounts of Kool-Aid alone and sugar water alone ( $>.05)$. As Figure 1 shows, there were no systematic changes in intakes over the conditioning period.

Although some of the animals that drank the alcohol solutions had difficulty in maintaining postural stability in the latter third of the 10-min drinking sessions, it did not prevent them from continuing to drink. However, it is possible that their rate of drinking may have been reduced.

The control animals self-administered an average dosage of $2.07 \mathrm{~g} / \mathrm{kg} /$ day, which was larger than the dosage of $1.51 \mathrm{~g} / \mathrm{kg}$ administered by the experimental animals $(\mathrm{F}=36.57, \mathrm{df}=1 / 18, \mathrm{p}<.01)$. This difference cannot be ascribed to weight differences because there were no significant weight differences between the groups before, during, or after the conditioning period.

The principal measure of conditioned aversion in this experiment involved between-group comparisons of postconditioning preference ratios, as well as within-group analyses of pre- and postconditioning ratios. A significant decrease in preference for the preferred flavor of Kool-Aid on postconditioning tests would be consistent with the conclusion that the presence of the alcohol in the Kool-Aid mixture resulted in that flavor's becoming secondarily aversive.

The results of the preconditioning and postconditioning preference tests are presented in Figure 2. A two-factor analysis of variance with repeated measures over the 2-day blocks of the postconditioning preference-testing period indicated that the experimental animals, which had the alcohol mixed with the preferred flavor of Kool-Aid during the conditioning period, drank less of the preferred flavor during the postconditioning sessions than did the controls for whom alcohol was never mixed with Kool-Aid $(\mathrm{F}=22.97, \mathrm{df}=1 / 18$, $\mathrm{p}<.01)$. Within-group analyses of the postconditioning 
preference tests indicated no significant changes over days in the drinking of either the control or the experimental group. A within-group comparison of the preconditioning with the first block of 2-day postconditioning preferences for the control animals demonstrated that there was no significant change in preference as a result of the procedures these animals were subjected to during the conditioning period. On the other hand, the experimental animals displayed a large decrease in preference as a result of the conditioning procedure $(\mathrm{F}=47.21, \mathrm{df}=1 / 9, \mathrm{p}<.01)$. It is of interest to note that all 10 experimental subjects demonstrated a decrease in preference. Furthermore, conditioned aversions were developed to the same extent for both grape $(\mathrm{N}=6)$ and orange $(\mathrm{N}=4)$ flavors.

The rank-order correlation between the mean amount of alcohol drunk per day during the conditioning period and the difference between the preconditioning and postconditioning preference ratios (first 2-day block) was not significantly different from zero (no $=-.43$ ).

\section{DISCUSSION}

Conditioned taste aversion was demonstrated in this experiment by significant differences in preference ratios between experimental and control groups on postconditioning preference tests and by within-group comparisons of pre- and postconditioning ratios. The experimental animals' significant decrease in preference for the preferred flavor of Kool-Aid on postconditioning tests is consistent with the conclusion that the presence of the alcohol in the Kool-Aid mixture during conditioning resulted in that flavor's becoming secondarily aversive. The conditioned aversion resulted from five presentations of Kool-Aid with 5\% alcohol at an average alcohol dosage of $1.51 \mathrm{~g} / \mathrm{kg} /$ day.

The controls had the same number of opportunities to drink the Kool-Aid and sugar water solution, but for them the 5\% alcohol was mixed with sugar water and never with Kool-Aid. Even though this procedure during the conditioning period resulted in the control animals' ingesting more alcohol than the experimental rats, there was no change in preference for the controls.

The consistently lower intake of solutions which contained alcohol relative to solutions without alcohol observed throughout the conditioning period could be due to unconditioned aversion to the alcohol solutions, and/or conditioned aversions, and/or a reduced rate of drinking in animals that ingested alcohol in sufficient quantities to become intoxicated prior to completion of the 10-min drinking period.

A learned taste-aversion interpretation would have predicted a progressive decrease in the amount of fluid consumed because it would be expected that repeated pairings would enhance the conditioned aversiveness of the alcohol solution and decrease the amount of that fluid consumed during subsequent sessions. The absence of progressive decreases in the consumption of the fluid mixed with alcohol during the forced-drinking conditioning period does not support such an interpretation, but it can perhaps be explained in terms of conflicting tendencies. Specifically, the forced-drinking procedure can be viewed as a conflict situation wherein the tendency to approach the drinking tube to obtain the daily fluid is opposed by a tendency to avoid the aversive alcohol solution. The lack of a progressive decrease in the consumption of either Kool-Aid + alcohol or sugar water + alcohol in the present experiment supports the conclusion that the relative strengths of approach and avoidance did not change during the conditioning period. Presumably, any increment in conditioned aversion was balanced out by comparable increases in the strength of the approach component. By contrast, Eckardt, Skurdal, and Brown (1974) found a progressive decrease in the amount consumed over conditioning days at dosages of $.8 \mathrm{~g} / \mathrm{kg}$ when administered IP, which is much less than the $1.51 \mathrm{~g} / \mathrm{kg}$ administered in this experiment. The latter observation is supportive of the hypothesis of Lester et al. (1970) that the administration of alcohol via IP or intracardiac catheter is more aversive than when introduced intragastrically.

The observations that the control animals self-administered significantly more alcohol than the experimentals during the conditioning period but did not change their preference, and that there were no changes in body weights during the conditioning period for either group, argue against the possibility that general malaise throughout the conditioning period served as the effective condition for the production of conditioned aversion. The results of this experiment provide no information on what is (are) the effective aversive component(s) but do indicate that the rapid drinking of $5 \%$ alcohol solutions has accompanying aversive consequences.

It may be noted that although the amounts of Kool-Aid consumed comprised the primary data evaluated in this experiment, in principle, the sugar water solution should also have become an aversive stimulus for the control animals since it was mixed with $5 \%$ alcohol during the conditioning period. No tests of this possibility were conducted.

\section{REFERENCES}

Berger, B. D. Conditioning of food aversions by injections of psychoactive drugs. Joumal of Comparative and Phy siological Psy chology, 1972, 81, 21-26.

Dragoin, W., McCleary, G. E., \& McCleary, P. A comparison of two methods of measuring conditioned taste aversions. Behavior Research Methods \& Instrumentation, 1971, 3, 309-310.

Eckandt, M. J., Skurdal, A. J., \& Brown, J. S. Conditioned taste aversion produced by low doses of alcohol. Physiological Psy chology, 1974, 2, 89-92.

Gillespie, R. J. G., \& Lucas, C. C. An unsuspected factor which influences consumption of alcohol by rats. Nature (Lond.), 1957, 180, $1292-1293$. 
Grote, F. W., \& Brown, R. T. Conditioned taste aversions: Two-stimulus tests are more sensitive than one-stimulus tests. Behavior Research Methods \& Instrumentation, 1971, 3, 311-312.

Kalant, H. Absorption, diffusion, distribution, and elimination of ethanol: Effects on biological membranes. In B. Kissin and H. Begleiter (Eds.), The biology of alcoholism: Volume 1 . New York: Plenum, 1971.

Lester, D. Self-selection of alcohol by animals, human variation, and the etiology of alcoholism: A critical review. Quarterly Journal of Studies on Alcohol, 1966, 27, 395-438.

Lester, D., Nachman, M., \& LeMagnen, J. Aversive conditioning by ethanol in the rat. Quarterly Journal of Studies on Alcohol, 1970, 31, 578-586.

Mardones, J. Experimentally induced changes in the free selection of ethanol. International Review of Neurobiology, $1960,2,41-76$
Myers, R. Voluntary alcohol consumption in animals: Peripheral and intracerebral factors. Psy chosomatic Medicine, 1966, 28. 484-497.

Myers, R., \& Veale, W. H. The determinants of alcohol preference in animals. In B. Kissin and H. Begleiter (Eds.). The biology of alcoholism: (Vol. 2). New York: Plenum, 1972.

Richter, C. P. Alcohol, beer, and wine as foods. Quarterly Journal of Studies on Alcohol, 1953, 14, 525-539.

(Received for publication December 19, 1974; revision accepted March 31,1975 .) 